\title{
SBR/Image Approach for Radio Wave Propagation in Furnished Environments
}

\author{
Shin-Hon Chen and Shyh-Kang Jeng* \\ Rm. 545, Department of Electrical Engineering, National Taiwan University \\ Taipei, Taiwan, Republic of China
}

\section{Introduction}

Detailed understanding of the radio propagation characteristics in a room will help the design of personal communication services (PCS) systems. In this paper, a deterministic approach using a modified shooting-and-bouncing-ray (SBR) technique [1] denoted as the $\mathrm{SBR}$ /image method is develped to deal with the radio wave propagation in furnished rooms, and the reception sphere is not needed[2][3][4]. Conceptually we use the SBR method to trace ray tubes (not rays) bouncing in the room. If the receiving antenna $(\mathrm{Rx})$ is within a ray tube, the ray tube will have contribution to the received field at $\mathrm{Rx}$ and the corresponding equivalent source (image) can be determined. In addition, the first order wedge diffraction from furniture is inctuded, and the diffracted rays also can be attributed to the corresponding images. By summing all contributions of these images, we can obtain the total received field at Rx. Numerical experiments have been conducted to investgate the radio wave propagation in rooms with metallic furniture, because these kinds of furniture are the most dominant reflectors in furnished rooms. The results show that the diffraction effect can be neglected in such environments where multiple reflection is severe.

\section{The SBR/Image Mețod}

To illustrate the SBR/image method, let us consider a room composed of triangular facets as in Fig.1. The notation $\mathrm{Tx}$ represents the transmitting antenna with a vector effective height (VEH) $\vec{h}_{t}[5][6]$ radiating in all directions in the room, and the notation $\mathrm{Rx}$ stands for the receiving antenna with a VEH $\vec{h}_{r}$. We shoot many ray tubes from Tx (Fig.2), and trace each ray tube which bounces in the room. For each bounce, we check whether the receiver falls within the reflected ray tube. If it does, the contribution of the ray tube to the receiver can be attributed to an equivalent source (image). In other words, a specular ray going to $\mathrm{Rx}$ exists in this tube, and this ray can be thought as launched from an image source. The image position, $\vec{\rho}_{o q}$ and the effective antenna height of the image $\vec{h}_{q}$ related to the $q$ 'th bounce of the refiected ray tube can be calculated by

$$
\vec{\rho}_{o q}=\vec{\rho}_{q}-\ell_{q} \cdot \hat{\zeta}_{q}
$$

and

$$
\vec{h}_{q}=\left(R_{\nu} \hat{e}_{\| \mid} \hat{e}_{\|\}}^{\prime}+R_{h} \hat{e}_{\perp} \hat{e}_{\perp}^{\prime}\right) \cdot \vec{h}_{q-1}
$$

respectively, where $\vec{\rho}_{q}$ is the reflection point of the center ray of the tube at the q'th bounce and $\ell_{q}$ is the path length from $T x$ to the reflection point. Symbols $\left(\hat{\zeta}_{q-1}, \hat{e}_{\|}^{\prime}, \hat{e}_{\perp}^{\prime}\right)$ and $\left(\hat{\zeta}_{q}, \hat{e}_{\|}, \hat{e}_{\perp}\right)$ are orthonormal bases, generated by the direction of incidence $\hat{\zeta}_{q-1}$ and the direction of 
reflection $\hat{\zeta}_{q}$, respectively(Fig.3). Notations $R_{h}$ and $R_{v}$ represent the reflection coefficients for horizontal and vertical polarizations, respectively. Using reciprocity theorem, the receiving amplitude $b 4$ [6] contributed by this image at $\mathrm{Rx}$ can be calculated. Repeat tracing the ray tube until the number of bounces is larger than the maximum number of bounces set beforehand. The square of the magnitude of the total b4 will equal the ratio of $P_{r}$ to $P_{t}$, where $P_{r}$ is the power received by $\mathrm{Rx}$ and $P_{t}$ is the total power transmitted in free space by Tx.

The field diffracted from illuminated wedges of furniture can be modeled by the Uniform Theory of Diffraction ( UTD ). Because the contribution of double diffraction is so small by our analysis that we only consider single diffraction in this paper. For every wedge of furniture, we check whether the diffracted point exists. If it does, the contribution of the diffracted ray to the receiver can be attributed to an equivalent source (image). In other words, this diffracted ray can be thought as launched from an image source.

\section{Numerical Results and Discussions}

In Fig.4, simulation results for different antenna orientations in a rectangular corridor with perfect electric conductor (PEC) walls are shown to verify our approach. The corridor is 4-meter wide and 4-meter high. Assume that the transmitting antenna with Idly operating at $1 \mathrm{GHz}$ is fixed at $(\mathrm{xt}, \mathrm{yt}, \mathrm{zt})=(1.1,2.1,0)$, while the receiver, another Herzian dipole, at $(\mathrm{xr}, \mathrm{yr}, \mathrm{zr})=(1.9,1.7, \mathrm{Zr})$ moves horizontally. The propagation loss $P_{r} / P_{t}$ with vertical (VVpolarization) and horizontal (VH-polarization) receiving antennas are shown in Figs.4(a) and (b), respectively. The solid line is computed from our SBR/Image method, and the dashed line is calculated by the exact modal solution [7]. It is seen that the agreement is very good.

In Fig.5(a), we consider a simply furnished room with a metallic closet which is $2 \mathrm{~m}$ high, $0.6 \mathrm{~m}$ wide and $7 \mathrm{~m}$ long. Half-wave dipole antenna $\mathrm{Tx}$ is located at $(\mathrm{xt}, \mathrm{yt}, \mathrm{zt})=(2,2.5,3)$, and the propagation loss $P_{r} / P_{t}$ received by $\mathrm{Rx}$ is computed along a 1.5 -meter-high path. In Fig.5(b), we observed that the diffracted fields from furniture has no significant effect on the numerical result even in the non line of sight (NLOS) region. The reason is given as the following: In such environments, the multiple reflection is severe and the reflected rays can go through the upper free region above the closet, and reach Rx. The contribution of such multiply-reffected rays is much higher than that of the diffracted rays arriving $\mathrm{Rx}$. In addition, the propagation loss in the empty room is also shown for comparison with this furnished room. They are very different with each other. This means that the multiple reflection effect of furniture in indoor wireless communication is significant.

In Fig.6, we applied our method to predict the propagation loss in a complex furnished room (Fig.1). The results show that the diffraction have no significant effects on the predicted results. In other words, The diffraction mechanism is minor and could be neglected in such indoor environments where multiple reflection is severe.

In summary, an SBR/Image approach to investigate the radio wave propagation in rooms has been proposed. It can provide a good model of the radio propagation inside a room. The 
concept of the reception sphere is not needed, and the results are not sensitive to the size of zach ray tube. This SBR/image method can be enhanced by including the wall penetration 3o that more complicated indoor environments will be handled in the near future. More results will be shown in our presentation.

\section{References}

[1] H. Ling, R. C. Chou and S. W. Lee, "Shooting and bouncing rays: calculating the RCS of an arbitrarily shaped cavity", IEEE Trans. Antennas and Propagation, vol.37, no.2, Feb.1989, pp.194-205.

[2] K. R. Schaubach, N. J. Davis IV and T. S. Rappaport, "A ray tracing method for predicting path loss and delay spread in microcellular environments" ,42nd IEEE Vehicular Technology Conference, May 1992, pp.932-935.

[3] S. Y. Seidel and T. S. Rappaport, "A ray tracing technique to predict path loss and delay spread inside room", Proc. IEEE GLOBECOM Conference, 1992, pp.649-653.

[4] W. Honcharenko, et. al., "Mechanisms governing UHF propagation on single floors in modern office rooms", IEEE Trans. on Vehicular Technology, vol.41, no.4. Nov.1992, pp.496-504.

[5] R.E. Collin, Antennas and Radiowave Propagation, McGraw-Hill, New York, 1985, chapter 5.

[6] Y.T. Lo and S.W. Lee ed., Antenna Handbook, New York: Van-Nostrand Reinhold, 1988, chapters 2,6

[7] C.T. Tai, Dyadic Green Functions, 2nd ed., IEEE Press, 1993, chapter 5.

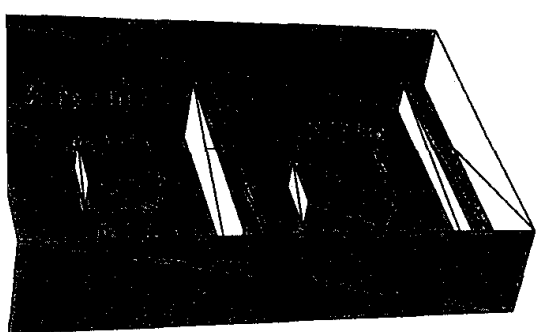

Fig. 1 A furnished room modeled by triangular facets.

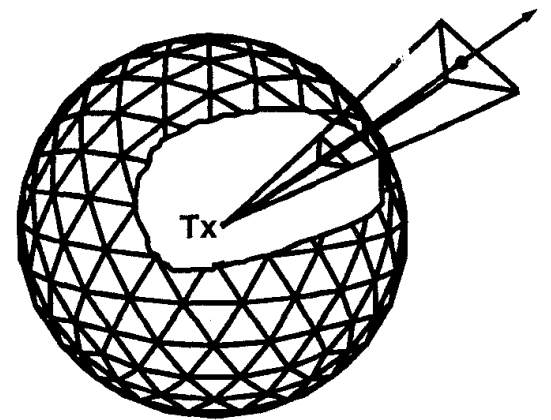

Fig. 2 Ray tubes shot from transmitting antenna $\mathrm{Tx}$. 


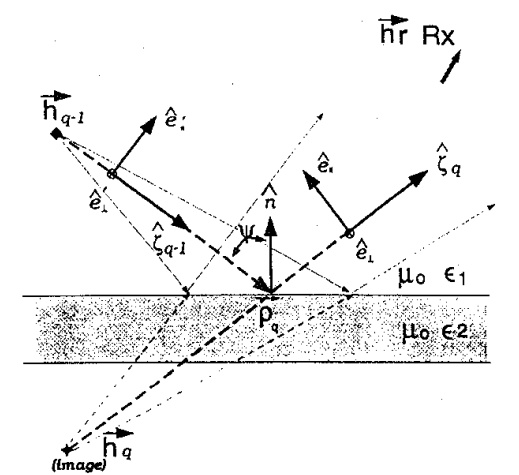

Fig. 3 Ray tube bounced at a planar interface, and image source having a contribution to the receiving antenna.
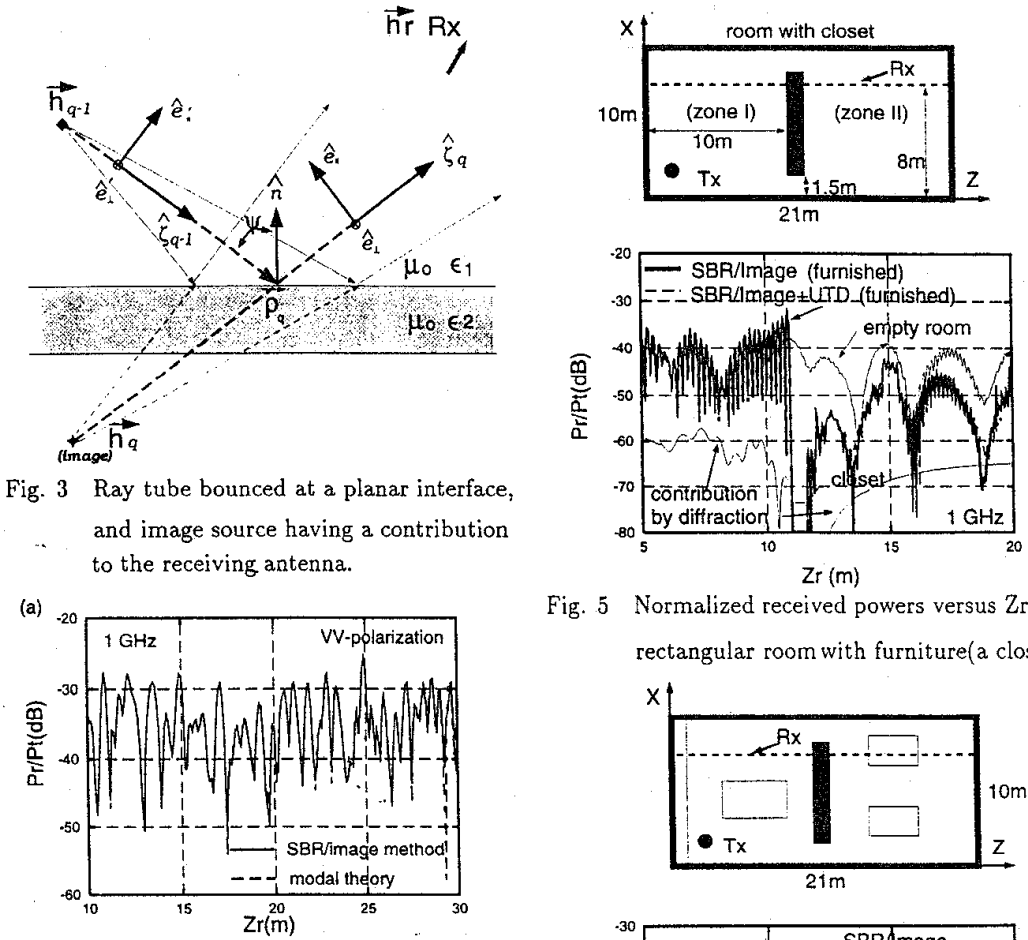

Fig. 5 Normalized received powers versus $\mathrm{Zr}$ in a rectangular room with furniture(a closet)
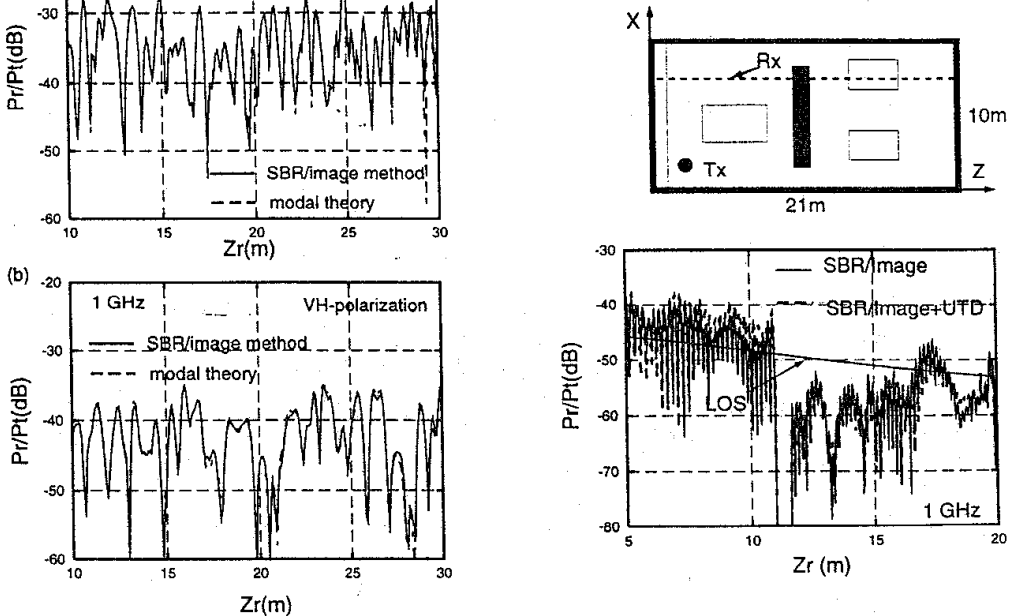

Fig. 4 Normalized received powers versus $\mathrm{Zr}$ in a rectangular corridor (a) VV-polarization. (b) VH-polarization.

Fig. 6 Normalized received powers versus $\mathrm{Zr}$ in a complex furnished room 